Pacific Journal of Mathematics

HOLOMORPHICALLY CONVEX COMPACT SETS AND 


\title{
HOLOMORPHICALLY CONVEX COMPACT SETS AND COHOMOLOGY
}

\author{
S. Trapani
}

\begin{abstract}
Conditions are given, on a domain $D$ of a Stein manifold $X$, for the cohomology groups $H^{q}(D ; \mathscr{F})$ to be Fréchet-Schwartz spaces for every $q \geq 0$ and every coherent sheaf $\mathscr{F}$ on $X$.
\end{abstract}

Introduction. Let $V$ be a complex space and $\mathscr{F}$ a coherent sheaf on $V$. It is well-known that we can endow $H^{q}(V ; \mathscr{F})$ of a structure of topological vector space such that its separated $\left.H^{q}(V ; \mathscr{F}) / \overline{0}\right)$ is a Fréchet-Schwartz (F.S.) space. It is of some interest to know when $H^{q}(V ; \mathscr{F})$ is itself F.S. (For instance it is possible, if the answer is affirmative, to prove a Künneth formula.) This is the case when $V$ is Stein and $\mathscr{F}$ is any coherent sheaf on $V$, or when $V=X-K$, where $X$ is Stein, $K$ is a compact set with a fundamental system of Stein neighborhoods and $\mathscr{F}$ is a coherent sheaf on $X$. This is proved in ([3], Théorème 2.19, page 40).

In this paper we find conditions on a domain $D$, in a connected Stein manifold $X$ of dimension $n>1$, which are sufficient for the groups $H^{q}(D ; \mathscr{F})$ to be F.S. and the cohomology groups with compact support $H_{K}^{q}(D ; \mathscr{F})$ to be D.F.S. (Dual of Fréchet-Schwartz) for every $q \geq 0$ and every coherent sheaf $\mathscr{F}$ on $X$. These conditions turn out to be also necessary if the complex dimension of $X$ is 2 . Also we obtain a cohomology duality theorem for such domains.

Preliminaries. Consider a domain $D$ in a connected Stein manifold $X$ of dimension $n>1$, let $S$ be the union of the connected compact components of $X-D$ and $D^{\prime}=D \cup S$; the set $D^{\prime}$ is open and connected ([11] page 30). Let $K$ be a compact subset of $X$ and $\mathscr{O}(K)$ be the direct limit $\lim _{U \supseteq K} \mathscr{O}(U)$ with the inductive limit topology; let $\operatorname{spec} \mathscr{O}(K)$ be the spectrum of $\mathscr{O}(K)$, i.e. the set of all nonzero continuous homomorphisms of the algebra $\mathscr{O}(K)$ into $\mathbf{C}$.

Following [13] we say that $K$ is holomorphically convex if the usual evaluation map $g: K \rightarrow \operatorname{spec} \mathscr{O}(K)$ given by $g(x)(f)=f(x)$ is bijective. 
In this paper we will study the domains $D$ which fulfill the following conditions:

(a) The domain $D^{\prime}$ is Stein.

(b) Every connected compact component of $X-D$ is a holomorphically convex compact set.

2. Cohomological properties. This section is dedicated to the study of the topology of the cohomology groups $H^{q}(D ; \mathscr{F})$ where $D$ is a domain in $X$ which fulfills conditions (a) and (b) and $\mathscr{F}$ is a coherent sheaf on $D^{\prime}$.

We will prove the following results:

THEOREM 1. If (a) and (b) hold for $D$ then:

(i) The space $H^{q}(D ; \mathscr{F})$ is $F . S$. for every $q \geq 0$ and every coherent sheaf $\mathscr{F}$ on $D^{\prime}$.

(ii) If $\mathscr{F}$ is locally free then $H^{q}(D ; \mathscr{F})=0$ for $0<q<n-1$.

TheOREM 2. If $D$ fulfills (a) and (b) and $\mathscr{F}$ is a coherent sheaf on $D^{\prime}$ then: the image of the restriction map $r: \Gamma\left(D^{\prime} ; \mathscr{F}\right) \rightarrow \Gamma(D ; \mathscr{F})$ is closed. If in addition prof $\mathscr{F} \geq 2$ then $r$ is bijective while if prof $\mathscr{F}=1$ then $r$ is injective.

TheOREM 3. A domain $D$ in $X$ fulfills conditions (a) and (b) if and only if $H^{q}(D ; \mathscr{O})=0$ for $0<q<n-1$ and $H^{n-1}(D ; \mathscr{O})$ is a F.S. space.

To prove these theorems we need some lemmata:

LEMMA 1. Let $0 \rightarrow X^{q} \stackrel{I}{\rightarrow} Y^{q} \stackrel{J}{\rightarrow} Z^{q} \rightarrow 0$ be a short exact sequence of complexes of F.S. or D.F.S. spaces and continuous linear maps; let:

$$
\cdots H^{q}(X) \stackrel{I^{q^{*}}}{\longrightarrow} H^{q}(Y) \stackrel{J^{q^{*}}}{\longrightarrow} H^{q}(Z) \stackrel{\delta^{q}}{\longrightarrow} H^{q+1}(X) \cdots
$$

be the cohomology exact sequence, then:

(1) The maps $I^{* q}, J^{* q}$ and $\delta^{q}$ are continuous.

(2) If one of these has a closed image then it is a topological homomorphism.

Proof. The maps $I^{* q}$ and $J^{* q}$ are obviously continuous. We denote with $Z^{q}(X) ; Z^{q}(Y)$ and $Z^{q}(Z)$ the spaces of cocycles and with $B^{q}(X)$; $B^{q}(Y)$ and $B^{q}(Z)$ the spaces of coboundaries. The maps $\pi_{X}^{q} ; \pi_{Y}^{q}$ and $\pi_{Z}^{q}$ will denote the projections:

$$
\begin{aligned}
& \pi_{X}^{q}: Z^{q}(X) \rightarrow H^{q}(X), \\
& \pi_{Y}^{q}: Z^{q}(Y) \rightarrow H^{q}(Y), \\
& \pi_{Z}^{q}: Z^{q}(Z) \rightarrow H^{q}(Z) .
\end{aligned}
$$


We have the commutative diagram:

$$
\begin{array}{crr}
Z^{q}(X) & \stackrel{I^{q}}{\longrightarrow} Z^{q}(Y) \\
\downarrow \pi_{X}^{q} & & \downarrow^{q} \\
H^{q}(X) & \stackrel{I^{* q}}{\longrightarrow} & H^{q}(Y) .
\end{array}
$$

If we suppose that $\operatorname{Im}\left(I^{* q}\right)=\pi_{Y}^{q}\left(\operatorname{Im}\left(I^{q}\right)\right)$ is closed, we get $\operatorname{Im} I^{q}+$ $B^{q}(Y)$ closed.

Let us take the map $\tau^{q}: Y^{q-1} \times Z^{q}(X) \rightarrow Z^{q}(Y)$ defined by $\tau^{q}(x, y)$ $=d_{Y}^{q+1}(x)+I^{q}(y)$ (where $d_{Y}^{q}$ is the coboundary map). Now $\tau^{q}$ is a map between F.S. or D.F.S. spaces and has a closed image; then it is a topological homomorphism. The map $\omega^{q}: Y^{q-1} \times Z^{q}(X) \rightarrow H^{q}(X)$ given by: $\omega^{q}(x ; y)=\pi_{X}^{q}(y)$ is onto and the diagram:

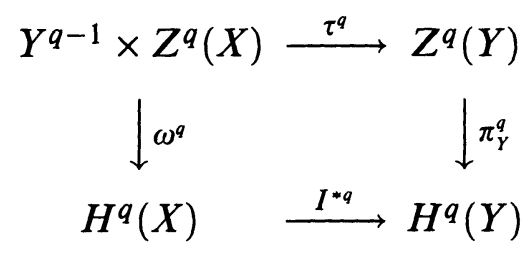

is commutative.

Since $\left.\pi^{q}\right|_{\operatorname{Im} I^{q}+B^{q}(Y)}$ is still a topological homomorphism, so is $I^{* q}$. The proof for $J^{* q}$ is the same.

We now look at $\delta^{q}$. To prove that $\delta^{q}$ is continuous is equivalent to proving that $\tilde{\delta}^{q}=\delta^{q} \circ \pi_{Z}^{q}: Z^{q}(Z) \rightarrow H^{q+1}(X)$ is. Now the surjective homomorphism, $I^{q}: Y^{q} \rightarrow Z^{q}$ is topological, then $J^{q}:\left(J^{q}\right)^{-1}\left(Z^{q}(Z)\right)$ $\rightarrow Z^{q}(Z)$ is topological onto and

$$
\tilde{J}^{q}: \frac{\left(J^{q}\right)^{-1}\left(Z^{q}(Z)\right)}{\operatorname{Ker} J^{q}} \rightarrow Z^{q}(Z)
$$

is a topological isomorphism. The coboundary map $d_{Y}^{q}$ is continuous and

$$
d_{Y}^{q}\left(\left(J^{q}\right)^{-1}\left(Z^{q}(Z)\right)\right) \subseteq \operatorname{Ker} J^{q+1}=\operatorname{Im} i^{q+1},
$$

and moreover $i^{q+1}$ is topological and injective; then the map $\sigma^{q}=$ $\left(i^{q+1}\right)^{-1} \circ d_{Y}^{q}$ is continuous.

We have that $\operatorname{Im} \sigma^{q} \subseteq Z^{q+1}(X)$, so we can define $\psi^{q}=\pi_{X}^{q+1} \circ \sigma^{q}$ and $\psi^{q}\left(\operatorname{Ker} J^{q}\right)=0$. The map

$$
\tilde{\psi}^{q}: \frac{\left(J^{q}\right)^{-1}\left(Z^{q}(Z)\right)}{\operatorname{Ker} J^{q}} \rightarrow H^{q+1}(X)
$$

is continuous then so is $\tilde{\delta}^{q}=\tilde{\psi}^{q} \circ\left(\tilde{J}^{q}\right)^{-1}$. 
Now $\delta^{q}$ is a topological homomorphism if and only if $\tilde{\delta}^{q}$ is, if and only if $\psi^{q}$ is.

We have $\operatorname{Im} \psi^{q}=\operatorname{Im} \delta^{q}$. So if $\operatorname{Im} \delta^{q}$ is closed we have $\pi_{X}^{q+1}\left(\operatorname{Im} \sigma^{q}\right)$ closed and $\operatorname{Im} \sigma^{q}+B^{q+1}(X)=\operatorname{Im} \sigma^{q}$ closed. Again $\sigma^{q}$ is topological and so are $\left.\pi_{X}^{q+1}\right|_{\operatorname{Im} \sigma^{q}}$ and $\psi^{q}$.

LeMma 2. Let $D$ be a domain in $X$, then there exists a locally finite partition of $S=D^{\prime}-D$ made by compact sets, say $\left\{K_{n}\right\}_{n \in \mathbf{N}}$. Let us put $K_{n}^{\prime}=\bigcup_{i=1}^{n} K_{i}$ and $D_{n}^{\prime}=D \cup K_{n}^{\prime}$, then:

(1) The set $D_{n}^{\prime}$ are domains.

(2) The domain D fulfills (b) if and only if each $K_{n}$ is holomorphically convex, in this case, each $K_{n}^{\prime}$ is holomorphically convex also.

Proof. The existence of a locally finite partition of $S$ by compact sets is proved in ([11] page 29). They are relatively open in $S$, then (2) follows from the fact that a compact set $K$ in $X$ is holomorphically convex if and only if every connected component of $K$ is ([15] page 14). (1) is proved in ([11] loc. cit. page 30).

LEMMA 3. Let $K$ be a holomorphically convex compact set on $X$ and $\mathscr{F}$ be a coherent analytic sheaf on $X$. Then the space $\Gamma(K ; \mathscr{F})=$ $\lim _{U \supseteq K} \Gamma(U ; \mathscr{F})$ with the inductive limit topology is D.F.S.

Proof. Let $\left\{U_{n}\right\}_{n \in \mathbf{N}}$ be a fundamental system of open neighborhoods of $K$ such that $U_{n+1} \subseteq U_{n}$.

For every $U_{n}$, let $\tilde{U}_{n}$ be the envelope of holomorphy of $U_{n}$. Each $\tilde{U}_{n}$ is thus a Riemann domain over $X$.

Let $\pi_{n}: \tilde{U}_{n} \rightarrow X$ be the projection on $X$; let $i_{n}: U_{n} \rightarrow \tilde{U}_{n}$ be the canonical embedding and for $n \geq m$ let $\phi_{n, m}: \tilde{U}_{n} \rightarrow \tilde{U}_{m}$ be the map induced by the inclusion $J_{n, m}: U_{n} \rightarrow U_{m}$.

Given the compact sets:

$$
\widehat{K}_{n}=\left\{x \in \tilde{U}_{n}:|f(x)| \leq \max _{K_{n}}|f| \forall f \in \mathscr{O}\left(\tilde{U}_{n}\right)\right\} .
$$

Consider the projective system $\left(\widehat{K}_{n} ; \phi_{n, m}\right)$; the projective limit of this system is isomorphic to $K$. Thus for every neighborhood $U_{n_{0}}$ there exists $n_{1} \geq n_{0}$ such that for every $n \geq n_{1}$ we have:

$$
\phi_{n_{0} ; n}\left(\widehat{K}_{n}\right) \subseteq i_{n_{0}}(K) \quad([13] \text { page 513). }
$$


Now if we take $\mathscr{F}_{n}^{*}$ on $\tilde{U}_{n}$ as the inverse image sheaf of $\mathscr{F}$ through $\pi$, we have an algebraic isomorphism:

$$
\underset{m \geq n}{\lim } \Gamma\left(\widehat{K}_{n} ; \mathscr{F}_{n}^{*}\right) \stackrel{\theta_{\mathscr{F}}}{\longrightarrow} \Gamma(K ; \mathscr{F}) .
$$

The map $\theta_{\mathscr{F}}$ derives from restriction maps and therefore it is continuous for the inductive limit topologies. Moreover $K$ is the projective limit of $\widehat{K}_{n}$ so every element in $\Gamma(K ; \mathscr{F})$ comes from an element in $\Gamma\left(\widehat{K}_{n} ; \mathscr{F}_{n}^{*}\right)$ for some $n \in N$. Then we have a continuous map:

$$
\rho: \Gamma(K ; \mathscr{F}) \rightarrow \underset{m \geq n}{\lim } \Gamma\left(\widehat{K}_{n} ; \mathscr{F}_{n}^{*}\right) .
$$

The map $\rho$ is the inverse of $\theta_{\mathscr{F}}$. The map $\theta_{\mathscr{F}}$ is therefore a topological isomorphism.

In a neighborhood of $K$ we have the exact sequence of sheaves:

$$
\begin{aligned}
& 0 \rightarrow \mathscr{G} \stackrel{\lambda}{\rightarrow} \mathscr{O}^{r} \stackrel{\mu}{\rightarrow} \mathscr{F} \rightarrow 0 \quad \text { and the corresponding: } \\
& 0 \rightarrow \Gamma\left(\widehat{K}_{n} ; \mathscr{G}_{n}^{*}\right) \stackrel{\lambda_{n}}{\longrightarrow} \Gamma\left(\widehat{K}_{n} ; \mathscr{O}_{n}^{* r}\right) \stackrel{\mu_{n}}{\rightarrow} \Gamma\left(\widehat{K}_{n} ; \mathscr{F}_{n}^{*}\right) \rightarrow 0, \\
& 0 \rightarrow \Gamma(K ; \mathscr{G}) \rightarrow \Gamma\left(K ; \mathscr{O}^{r}\right) \rightarrow \Gamma(K ; \mathscr{F}) \rightarrow 0 .
\end{aligned}
$$

In fact for holomorphically convex compact sets, theorem B holds ([6] page 73).

The spaces $\Gamma\left(\widehat{K}_{n} ; \mathscr{G}_{n}^{*}\right), \Gamma\left(\widehat{K}_{n} ; \mathscr{O}_{n}^{r^{*}}\right)$, and $\Gamma\left(\widehat{K}_{n} ; \mathscr{F}_{n}^{*}\right)$ with the inductive limit topology are D.F.S. and the maps of the exact sequences are topological homomorphisms ([2] page 236).

If we pass to the inductive limit we get:

$$
\begin{aligned}
& 0 \rightarrow \underset{n}{\lim } \Gamma\left(\widehat{K}_{n} ; \mathscr{G}_{n}^{*}\right) \stackrel{\tilde{\lambda}}{\longrightarrow} \underset{n}{\lim } \Gamma\left(\widehat{K}_{n} ; \mathscr{O}_{n}^{r^{*}}\right) \stackrel{\tilde{\mu}}{\longrightarrow} \underset{n}{\lim } \Gamma\left(\widehat{K}_{n} ; \mathscr{F}_{n}^{*}\right) \rightarrow 0 \\
& \downarrow \theta_{\mathscr{G}} \quad \downarrow \theta_{\text {Or }} \quad \downarrow \theta_{\mathscr{F}} \\
& 0 \rightarrow \Gamma(K ; \mathscr{G}) \longrightarrow \Gamma\left(K ; \mathscr{O}^{r}\right) \longrightarrow \Gamma(K ; \mathscr{F}) \rightarrow 0 .
\end{aligned}
$$

The space $\Gamma\left(K ; \mathscr{O}^{r}\right)$ is D.F.S. ([2] page 234) and, $\theta_{\mathscr{O}^{r}}$ being topological isomorphism, $\lim _{n} \Gamma\left(\widehat{K}_{n} ; \mathscr{O}_{n}^{r^{*}}\right)$ is D.F.S. also. Since topological homomorphisms are preserved by passing to denumerable inductive limit, ([2] page 227) the maps $\tilde{\lambda}$ and $\tilde{\mu}$ are topological. It follows that $\lim _{n} \Gamma\left(\widehat{K}_{n} ; \mathscr{G}^{*}\right)$ is a Hausdorff space, so it is D.F.S. and its image through $\tilde{\lambda}$ is closed. Hence $\lim _{n} \Gamma\left(\widehat{K}_{n} ; \mathscr{F}^{*}\right)$ is D.F.S.

Let $U$ be an open neighborhood of a compact set $K \subseteq X$ and $\mathscr{F}$ a coherent sheaf on $U$. 
We have the exact sequences:

$$
\begin{aligned}
& \rightarrow H_{K}^{q}(U ; \mathscr{F}) \stackrel{I_{\mathscr{F}}^{q}}{\longrightarrow} H^{q}(U ; \mathscr{F}) \stackrel{r_{\mathscr{F}}^{q}}{\longrightarrow} H^{q}(U-K ; \mathscr{F}) \\
& \stackrel{\delta_{\mathscr{F}}^{q}}{\longrightarrow} H_{K}^{q+1}(U ; \mathscr{F}) \rightarrow \cdots \\
& \rightarrow \operatorname{Ext}_{k}^{q}\left(U-K ; \mathscr{F} ; \Omega^{n}\right) \stackrel{I_{\mathscr{G}}}{\longrightarrow} \operatorname{Ext}_{k}^{q}\left(U ; \mathscr{F} ; \Omega^{n}\right) \\
& \stackrel{r_{\mathscr{F} q}}{\longrightarrow} \operatorname{Ext}^{q}\left(K ; \mathscr{F} ; \Omega^{n}\right) \stackrel{\delta_{\mathscr{F}_{q}}}{\longrightarrow} \operatorname{Ext}_{k}^{q+1}\left(U-K ; \mathscr{F} ; \Omega^{n}\right) \rightarrow \cdots
\end{aligned}
$$

( $\Omega^{n}$ is the sheaf of holomorphic differential $n$ forms).

There exist two short exact sequences of F.S. respectively D.F.S. spaces, and continuous linear maps, whose cohomology sequences are (2.2) and (2.3) respectively. If we consider on (2.2) and (2.3) the induced topologies, then the sequences of the separated spaces are dual to each other. Moreover:

\section{D.F.S.}

$(\alpha) H^{q}(U-K ; \mathscr{F})$ is F.S. if and only if $\operatorname{Ext}_{k}^{n-q+1}\left(U-K ; \mathscr{F} ; \Omega^{n}\right)$ is

( $\beta) H^{q}(U ; \mathscr{F})$ is F.S. if and only if $\operatorname{Ext}_{k}^{n-q+1}\left(U ; \mathscr{F} ; \Omega^{n}\right)$ is D.F.S.

(y) $H_{K}^{q}(U ; \mathscr{F})$ is F.S. if and only if $\operatorname{Ext}^{n-q+1}\left(K ; \mathscr{F} ; \Omega^{n}\right)$ is D.F.S. ([1] page 1163).

We now can prove the following:

LEMMA 4. If $U$ is Stein and $K$ is holomorphically convex, then the topology on $\operatorname{Hom}\left(K ; \mathscr{F} ; \Omega^{n}\right)$ of the sequence (2.3) is D.F.S. and coincides with the inductive limit topology of $\Gamma\left(W ; \operatorname{Hom}\left(\mathscr{F} ; \Omega^{n}\right)\right)$, where $W$ runs in a fundamental system of open neighborhoods of $K$.

Proof. We have that $H^{n}(U-K ; \mathscr{F})=H^{n+1}(U ; \mathscr{F})=0$ [9]. Then $H_{K}^{n+1}(U ; \mathscr{F})=0$ and by $(\gamma)$ the space $\operatorname{Hom}\left(K ; \mathscr{F} ; \Omega^{n}\right)$ with the topology of (2.3) is D.F.S.

Denote this topology by $T$.

Note that if $W$ is another Stein neighborhood of $K$ contained in $U$ then, by excision the $T$ topology on $\operatorname{Hom}\left(K ; \mathscr{F} ; \Omega^{n}\right)$ with respect to $W$ equals that with respect to $U$.

On the other hand, by Lemma $3, \operatorname{Hom}\left(K ; \mathscr{F} ; \Omega^{n}\right)$ with the inductive limit topology is D.F.S.

Denote this topology by $L$. We must prove that $L$ and $T$ topologies coincide.

First step: $\mathscr{F}=\mathscr{O}$. Let $W$ be an open neighborhood of $K$ in $U$ and $J_{W}: H_{K}^{n}(U ; \mathscr{O}) \rightarrow H_{K}^{n}(W ; \mathscr{O})$ the inclusion map. The map 
$J_{W} \circ \delta^{n-1}: H^{n-1}(U-K ; \mathscr{O}) \rightarrow H_{K}^{n}(W ; \mathscr{O})$ is continuous for the standard topologies. This is proved in [3] pages $42-43$ when $K$ has a fundamental system of Stein neighborhoods; however the proof in the general case is the same. Recall now that $H^{n}(U-K ; \mathscr{O})=0$ and by $(\alpha)$ that $\operatorname{Ext}_{k}^{1}\left(U-K ; \mathscr{O} ; \Omega^{n}\right)$ is D.F.S. We can conclude that the dual map of

$$
\lim _{W \supseteq K}\left(J_{W} \circ \delta^{n-1}\right): H^{n-1}(U-K ; \mathscr{O}) \rightarrow \underset{W \supseteq K}{\lim _{W}} H_{k}^{n}(W ; \mathscr{O})
$$

can be identified with $\delta_{0}: \operatorname{Hom}\left(K ; \mathscr{O} ; \Omega^{n}\right) \rightarrow \operatorname{Ext}_{k}^{1}\left(U-K ; \mathscr{O} ; \Omega^{n}\right)$, where $\operatorname{Hom}\left(K ; \mathscr{O} ; \Omega^{n}\right)$ has the $L$ topology and $\operatorname{Ext}_{k}^{1}\left(U-K ; \mathscr{O} ; \Omega^{n}\right)$ has its standard one. With these topologies $\delta_{0}$ is a continuous map between D.F.S. spaces. Since $U$ is $\operatorname{Stein}, \operatorname{Hom}_{k}\left(U ; \mathscr{O} ; \Omega^{n}\right)$ $=\operatorname{Ext}_{k}^{1}\left(U ; \mathscr{O} ; \Omega^{n}\right)=0$ and $\delta_{0}$ is bijective. Therefore $\delta_{0}$ is a topological isomorphism. By Lemma $1, \delta_{0}$ is a topological isomorphism also if $\operatorname{Hom}\left(K ; \mathscr{O} ; \Omega^{n}\right)$ has the $T$ topology and $\operatorname{Ext}_{k}^{1}\left(U-K ; \mathscr{O} ; \Omega^{n}\right)$ has its standard one. So $L$ and $T$ coincide on $\operatorname{Hom}\left(K ; \mathscr{O} ; \Omega^{n}\right)$.

In the general case, if $\mathscr{F}$ is coherent on $U$, choose a sequence like (2.1): on an open Stein neighborhood $W$ of $K$ in $U$. From this we derive:

$$
0 \rightarrow \operatorname{Hom}\left(\mathscr{F} ; \Omega^{n}\right) \stackrel{\mu^{\prime}}{\rightarrow} \operatorname{Hom}\left(\mathscr{O}^{r} ; \Omega^{n}\right) \stackrel{\lambda^{\prime}}{\rightarrow} \operatorname{Im} \lambda^{\prime} \rightarrow 0
$$

and

$$
\begin{aligned}
& 0 \rightarrow \operatorname{Hom}\left(K ; \mathscr{F} ; \Omega^{n}\right) \stackrel{\mu^{\prime *}}{\longrightarrow} \operatorname{Hom}\left(K ; \mathscr{O}^{r} ; \Omega^{n}\right) \\
& \quad \stackrel{\lambda^{\prime *}}{\longrightarrow} \Gamma\left(K ; \operatorname{Im} \lambda^{\prime}\right) \rightarrow \cdots
\end{aligned}
$$

We have the commutative diagram:

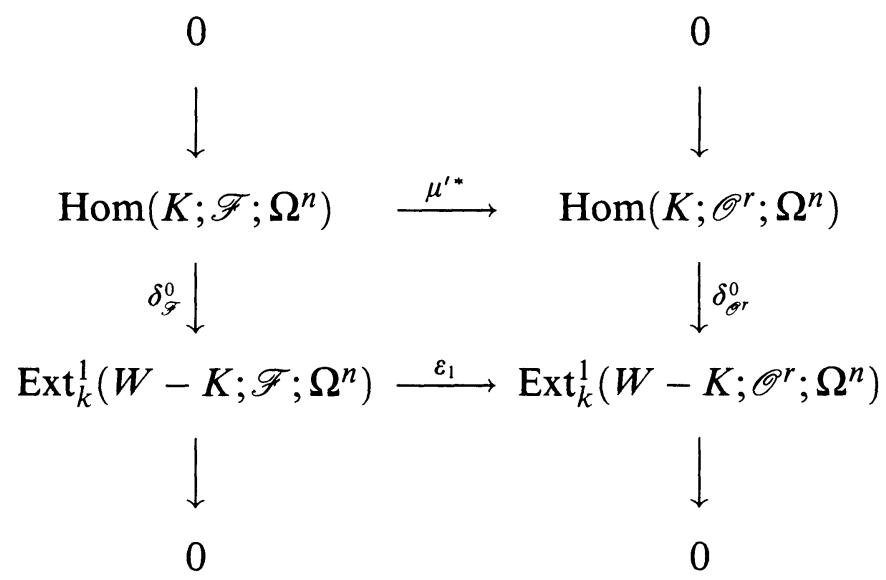


where $\varepsilon_{1}$ comes from the sequence:

$$
\begin{aligned}
& \rightarrow \operatorname{Ext}_{k}^{q}\left(W-K ; \mathscr{F} ; \Omega^{n}\right) \stackrel{\varepsilon_{q}}{\longrightarrow} \operatorname{Ext}_{k}^{q}\left(W ; \mathscr{F} ; \Omega^{n}\right) \\
& \rightarrow \operatorname{Ext}^{q}\left(K ; \mathscr{F} ; \Omega^{n}\right) \stackrel{\theta_{q}}{\longrightarrow} \operatorname{Ext}_{k}^{q+1}\left(W-K ; \mathscr{F} ; \Omega^{n}\right) \rightarrow \cdots .
\end{aligned}
$$

The map $\mu^{\prime *}$ is obviously $L$ continuous and $\Gamma\left(K ; \operatorname{Im} \lambda^{\prime}\right)$ with the $L$ topology is D.F.S., it follows that $\operatorname{Im} \mu^{\prime *}$ is $L$ closed. So it is an injective $L$ topological homomorphism. From the diagram (2.5) we obtain that $\mu^{\prime *}$ is $T$ continuous also and has $L$ closed image, therefore, by the first step, it has $T$ closed image and it is a $T$ topological homomorphism.

Proof of Theorem 1 Given $\mathscr{F}$ coherent on $D^{\prime}$ we have that $H^{0}(D ; \mathscr{F})$ is F.S. and $H^{n}(D ; \mathscr{F})=0$, so we must prove that $H^{q}(D ; \mathscr{F})$ is F.S. for $0<q \leq n-1$.

First step: $S$ compact, $\mathscr{F}$ locally free:

Let us choose a Stein neighborhood $U$ of $S$, since $S$ is holomorphically convex and $U$ is $\operatorname{Stein,} \operatorname{Ext}^{q}\left(S ; \mathscr{F} ; \Omega^{n}\right)=H^{q}\left(S ; \operatorname{Hom}\left(\mathscr{F} ; \Omega^{n}\right)\right)=$ 0 if $q>0$ and $\operatorname{Ext}_{k}^{q}\left(U ; \mathscr{F} ; \Omega^{n}\right)=0$ if $q \neq 0$ and $q \neq n$ while $\operatorname{Ext}_{k}^{n}\left(U ; \mathscr{F} ; \Omega^{n}\right)$ is D.F.S.

It follows, using the sequence $(2.3)$ that $\operatorname{Ext}_{k}^{q}\left(U-S ; \mathscr{F} ; \Omega^{n}\right)=0$ if $q \neq 0$ and $q \neq n$ while $\operatorname{Ext}_{k}^{n}\left(U-S ; \mathscr{F} ; \Omega^{n}\right)$ is D.F.S. Applying $(\alpha)$ we derive:

$H^{q}(U-S ; \mathscr{F})=0$ if $0<q<n-1$ and $H^{n-1}(U-S ; \mathscr{F})$, is F.S.

Second step: $S$ compact, $\mathscr{F}$ coherent:

The first step proves our statement if $\mathscr{F}$ is locally free, i.e. prof $\mathscr{F}=$ $n$. The second step follows by induction on $S(\mathscr{F})=n-\operatorname{prof} \mathscr{F}$. In fact: let us choose a sequence like (2.1): $0 \rightarrow \mathscr{G} \rightarrow \mathscr{O}^{r} \rightarrow \mathscr{F} \rightarrow 0$, on a Stein neighborhood $W$ of $S$ in $U$, where $S(\mathscr{G}) \leq S(\mathscr{F})-1$.

(Such a sequence exists by [3] page 243.)

It induces the cohomology sequence:

$$
\cdots \rightarrow H^{q}\left(W-S ; \mathscr{O}^{r}\right) \rightarrow H^{q}(W-S ; \mathscr{F}) \rightarrow H^{q+1}(W-S ; \mathscr{G}) \rightarrow \cdots
$$

if $0<q<n-1$, then $H^{q}\left(W-S ; \mathscr{O}^{r}\right)=0$ and $H^{q+1}(W-S ; \mathscr{G})$ is F.S. By induction hypothesis, so $H^{q}(W-S ; \mathscr{F})$ is F.S. and by excision $H^{q}(U-S ; \mathscr{F})$ is F.S.

If $q=n-1$ the cohomology sequence is:

$$
\begin{aligned}
\cdots & \rightarrow H^{n-1}(W-S ; \mathscr{G}) \stackrel{\tilde{\lambda}^{n-1}}{\longrightarrow} H^{n-1}\left(W-S ; \mathscr{O}^{r}\right) \\
& \stackrel{\tilde{\mu}^{n-1}}{\longrightarrow} H^{n-1}(W-S ; \mathscr{F}) \rightarrow 0 .
\end{aligned}
$$


By Lemma $1, \tilde{\mu}^{n-1}$ is a topological homomorphism onto, hence $H^{n-1}(W-S ; \mathscr{F})$ is F.S. if and only if $\operatorname{Im} \tilde{\lambda}^{n-1}$ is closed. By induction hypotheses $H^{n-1}(W-S ; \mathscr{G})$ and $H^{n-1}\left(W-S ; \mathscr{O}^{r}\right)$ are F.S., so $\operatorname{Im} \tilde{\lambda}^{n-1}$ is closed if and only if $\tilde{\lambda}$ is topological. The spaces $\operatorname{Ext}_{k}^{1}(W-S ; \mathscr{G})$ and $\operatorname{Ext}_{k}^{1}\left(W-S ; \mathscr{O}^{r}\right)$ are D.F.S. and the dual map of $\tilde{\lambda}^{n-1}$ and be identified with the $\varepsilon_{1}$ of the sequence (2.6). Then we must prove that $\varepsilon_{1}$ is topological or, which is the same, that it has closed image. From the commutative diagram (2.5) it follows that we need to prove that $\operatorname{Im} \mu^{\prime *}$ is $T$ closed. This has been shown while proving Lemma 4.

Hence $H^{n-1}(W-S ; \mathscr{F})$ if F.S. and again by excision $H^{n-1}(U-S ; \mathscr{F})$ is F.S. If we choose $U=D^{\prime}$ we conclude the proof in case $S$ is compact.

Third step: $S$ is not compact, $\mathscr{F}$ is coherent.

Let $\left\{K_{n}\right\}_{n \in \mathbf{N}}$ be a locally finite partition of $S$ by holomorphically convex compact sets as in Lemma 2.

Let $\left\{U_{n}\right\}_{n \in \mathbf{N}}$ be a sequence of disjoint open neighborhoods of $K_{n}$, and $U=\bigcup_{n} U_{n}$. We have for $q>0$ the topological isomorphisms:

$$
\begin{aligned}
H^{q}(D ; \mathscr{F}) & \sim H_{S}^{q+1}\left(D^{\prime} ; \mathscr{F}\right) \sim H_{S}^{q+1}(U ; \mathscr{F}) \sim \prod_{n} H_{K_{n}}^{q+1}\left(U_{n} ; \mathscr{F}\right) \\
& \sim \prod_{n} H_{K_{n}}^{q+1}\left(D^{\prime} ; \mathscr{F}\right) \sim \prod_{n} H^{q}\left(D^{\prime}-K_{n} ; \mathscr{F}\right) .
\end{aligned}
$$

By the second step $H^{q}\left(D^{\prime}-K_{n} ; \mathscr{F}\right)$ is F.S. for every $n \in \mathbf{N}$, further $H^{q}\left(D^{\prime}-K_{n} ; \mathscr{F}\right)=0$ if $\mathscr{F}$ is locally free and $0<q<n-1$.

\section{Proof of Theorem 2.}

First step: $S$ compact.

Let $\mathscr{F}$ be a coherent sheaf on $D^{\prime}$, since theorem $B$ holds for $S$, there is a canonical isomorphism

$$
\operatorname{Ext}^{q}\left(S ; \mathscr{F} ; \Omega^{n}\right) \rightarrow \Gamma\left(S ; \operatorname{Ext}^{q}\left(\mathscr{F} ; \Omega^{n}\right)\right) \text { for every } q \geq 0 .
$$

\section{([3] page 35.)}

If prof $\mathscr{F} \geq 2$ then $\operatorname{Ext}^{n}\left(\mathscr{F} ; \Omega^{n}\right)=\operatorname{Ext}^{n-1}\left(\mathscr{F} ; \Omega^{n}\right)=\operatorname{Ext}^{n}\left(S ; \mathscr{F} ; \Omega^{n}\right)$ $=\operatorname{Ext}^{n-1}\left(S ; \mathscr{F} ; \Omega^{n}\right)=0$ and $H_{S}^{0}\left(D^{\prime} ; \mathscr{F}\right)=H_{S}^{1}\left(D^{\prime} ; \mathscr{F}\right)=0$. Hence the restriction map $r: \Gamma(D ; \mathscr{F}) \rightarrow \Gamma(D ; \mathscr{F})$ is bijective. If prof $\mathscr{F}=1$ then $\operatorname{Ext}^{n}\left(\mathscr{F} ; \Omega^{n}\right)=\operatorname{Ext}^{n}\left(S ; \mathscr{F} ; \Omega^{n}\right)=0$, so $H_{S}^{0}(D ; \mathscr{F})=0$ and $H_{S}^{1}(D ; \mathscr{F})$ is F.S. by $(\gamma)$.

Hence the restriction is injective and has closed image.

If prof $\mathscr{F}=0$, then there exists a short exact sequence:

$$
0 \rightarrow \mathscr{H}_{A}^{0}(\mathscr{F}) \stackrel{h}{\rightarrow} \mathscr{F} \rightarrow \mathscr{G} \stackrel{l}{\rightarrow} 0 \text { on } D^{\prime} \text { such that: }
$$


$\left.\mathscr{H}_{A}^{0}(\mathscr{F})\right|_{D^{\prime}-A}=0$, the set $A$ is discrete on $D^{\prime}$ and prof $\mathscr{G} \geq 1$. So we derive the commutative diagram:

$$
\begin{array}{ccc}
0 \rightarrow \Gamma\left(D^{\prime} ; \mathscr{H}_{A}^{0}(\mathscr{F}) \stackrel{h_{D^{\prime}}}{\longrightarrow} \Gamma\left(D^{\prime} ; \mathscr{F}\right) \stackrel{l_{D^{\prime}}}{\longrightarrow} \Gamma\left(D^{\prime} ; \mathscr{G}\right) \rightarrow 0\right. \\
\qquad\left.\right|_{\mathscr{X}_{A}^{0}(\mathscr{F})} & \left.\right|_{\mathscr{F}} & \downarrow_{\mathscr{G}} \\
0 \rightarrow \Gamma\left(D ; \mathscr{H}_{A}^{0}(\mathscr{F})\right) \stackrel{h_{D}}{\longrightarrow} \Gamma(D ; \mathscr{F}) \stackrel{l_{D}}{\longrightarrow} \Gamma(D ; \mathscr{G}) \rightarrow 0 .
\end{array}
$$

In fact $H^{1}\left(D^{\prime} ; \mathscr{H}_{A}^{0}(\mathscr{F})\right)=H^{1}\left(D ; \mathscr{H}_{A}^{0}(\mathscr{F})\right)$ since $\mathscr{H}_{A}^{0}(\mathscr{F})$ has discrete support.

The map $r_{\mathscr{H}_{A}^{0}}$ is onto (every section on $\Gamma\left(D ; \mathscr{H}_{A}^{0}(\mathscr{F})\right.$ ) can be extended to $D^{\prime}$ as 0 ); hence $\operatorname{Im} r_{\mathscr{F}} \supseteq \operatorname{Im} h_{D} \circ r_{\mathscr{P}_{D}^{0}(\mathscr{F})}=\operatorname{Im} h_{D}=\operatorname{Ker} l_{D}$. So $\operatorname{Im} r_{\mathscr{F}}=l_{D}^{-1}\left(\operatorname{Im} l_{D} \circ r_{\mathscr{F}}\right)=l_{D}^{-1} \operatorname{Im}\left(r_{\mathscr{G}} \circ l_{D^{\prime}}\right)=l_{D}^{-1}\left(\operatorname{Im} r_{\mathscr{G}}\right)$. Now prof $\mathscr{G} \geq$ 1 , therefore $\operatorname{Im} r_{\mathscr{G}}$ is closed and so is $\operatorname{Im} r_{\mathscr{F}}$. The space $H_{S}^{1}\left(D^{\prime} ; \mathscr{F}\right)$ is then F.S.

Second step: $S$ is noncompact.

Let $\left\{K_{n}\right\}_{n \in \mathbf{N}}$ be a locally finite partition of $S$ by holomorphically convex compact sets. We obtain, as already observed in the proof of Theorem 1 , that $H_{S}^{q}\left(D^{\prime} ; \mathscr{F}\right) \sim \prod_{n} H_{K_{n}}^{q}\left(D^{\prime} ; \mathscr{F}\right)$ for every $q \geq 0$.

Proof of Theorem 3. If $D$ fulfills (a) and (b) then $H^{q}(D ; \mathscr{O})=0$ for $0<q<n-1$ and $H^{n-1}(D ; \mathscr{O})$ is F.S. by Theorem 1 .

Conversely, choose a locally finite partition $\left\{K_{r}\right\}_{r \in \mathbf{N}}$ of $S$ by compact sets. Let $K_{r}^{\prime}$ and $D_{r}^{\prime}$ be defined as in Lemma 2. Consider the Mayer-Vietoris sequence of the pair $\left(D_{r}^{\prime} ; X-K_{r}^{\prime}\right)$ :

$$
\begin{aligned}
\cdots & \rightarrow H^{q}(X ; \mathscr{O}) \rightarrow H^{q}\left(D_{r}^{\prime} ; \mathscr{O}\right) \times H^{q}\left(X-K_{r}^{\prime} ; \mathscr{O}\right) \rightarrow H^{q}(D ; \mathscr{O}) \\
& \rightarrow H^{q+1}(X ; \mathscr{O}) \rightarrow \cdots .
\end{aligned}
$$

From the hypotheses we deduce that $H^{q}\left(D_{r}^{\prime} ; \mathscr{O}\right)=0$ if $0<q<n-1$ while $H^{n-1}\left(D_{r}^{\prime} ; \mathscr{O}\right)$ is F.S.

But $D_{r}^{\prime} \subseteq D_{r+1}^{\prime}$ and $D^{\prime}=\bigcup_{r \in \mathbf{N}} D_{r}^{\prime}$. By the Hartogs theorem the restrictions $r^{q}: H^{q}\left(D_{r+1}^{\prime} ; \mathscr{O}\right) \rightarrow H^{q}\left(D_{r}^{\prime} ; \mathscr{O}\right)$ are bijective for $r \in \mathbf{N}$ and $0 \leq q<n-1$. It follows that $H^{q}\left(D^{\prime} ; \mathscr{O}\right)=\lim _{r} H^{q}\left(D_{r}^{\prime} ; \mathscr{O}\right)$ for $0<$

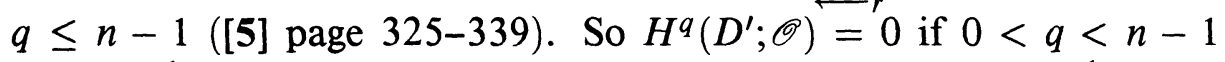
and $H^{n-1}\left(D^{\prime} ; \mathscr{O}\right)$ is F.S. To prove (a) we must prove that $H^{n-1}\left(D^{\prime} ; \mathscr{O}\right)$ vanishes or, which is the same, that its dual vanishes. By the Serre duality theorem $\left[H^{n-1}(D ; \mathscr{O})\right]^{\prime} \sim H_{k}^{1}\left(D^{\prime} ; \Omega^{n}\right)$. From the sequence:

$$
\begin{aligned}
& 0 \rightarrow \Omega_{X-D^{\prime}}^{n} \rightarrow \Omega^{n} \rightarrow \Omega_{D^{\prime}}^{n} \rightarrow 0 \quad \text { we derive : } \\
& 0 \rightarrow \Gamma_{k}\left(X-D^{\prime} ; \Omega^{n}\right) \rightarrow H_{k}^{1}\left(D^{\prime} ; \Omega^{n}\right) \rightarrow H_{k}^{1}\left(X ; \Omega^{n}\right) \rightarrow \cdots .
\end{aligned}
$$


So $H_{k}^{1}\left(D^{\prime} ; \Omega^{n}\right) \sim \Gamma_{k}\left(X-D^{\prime} ; \Omega^{n}\right)$. By construction the set $X-D^{\prime}$ has no compact connected components, then $\Gamma_{k}\left(X-D^{\prime} ; \Omega^{n}\right)=0$ and (a) follows.

To prove (b), fix $r \in \mathbf{N}$, then (2.8) gives that $H^{q}\left(X-K_{r}^{\prime} ; \mathscr{O}\right)=0$ for $0<q<n-1$ and $H^{n-1}\left(X-K_{r}^{\prime} ; \mathscr{O}\right)$ is F.S. Then $H_{k}^{q}\left(X-K_{r}^{\prime} ; \Omega^{n}\right)=0$ for $q \neq 1$ and $q \neq n$ while $H_{k}^{1}\left(X-K_{r}^{\prime} ; \Omega^{n}\right)$ and $H_{k}^{n}\left(X-K_{r}^{\prime} ; \Omega^{n}\right)$ are D.F.S. From the cohomology sequence with compact support of: $0 \rightarrow \Omega_{X-K_{r}^{\prime}}^{n} \rightarrow \Omega^{n} \rightarrow \Omega_{K_{r}^{\prime}}^{n} \rightarrow 0$, from duality and from the Hartogs theorem we conclude that $H^{q}\left(K_{r}^{\prime} ; \Omega^{n}\right)=0$ for every $q>0$. Let $E$ be the vector bundle of holomorphic differential $n$-forms. The manifold $X$ is Stein, then for every $p \in K_{r}^{\prime}$ there exists $\omega_{p} \in \Gamma(X ; E)$ with $\omega_{p}(p) \neq 0$. By compactness we can choose a finite number of sections $\omega_{1}, \ldots, \omega_{m}$ such that $\bigcap_{m}\left\{x \in X: \omega_{m}(x)=0\right\} \cap K_{r}^{\prime}=\varnothing$. So there exists an open neighborhood $U$ of $K_{r}^{\prime}$ and a nowhere zero section on $U$ of the bundle

$$
E^{m}=\underbrace{E \oplus E \cdots \oplus E}_{m \text { times }}
$$

Hence $\left.E^{m}\right|_{U}$ has a trivial subbundle $F$ of rank 1. Choosing a Hermitian metric on $\left.E^{m}\right|_{U}$ we can construct a subbundle $G$ of $\left.E^{m}\right|_{U}$ such that $\left.E^{m}\right|_{U}=G \oplus F$. If $\mathscr{G}$ denotes the sheaf of sections of $G$ we have for $q>0$ :

$$
\begin{aligned}
0 & =\left(H^{q}\left(K_{r}^{\prime} ; \Omega^{n}\right)\right)^{m}=H^{q}\left(K_{r}^{\prime} ;\left(\Omega^{n}\right)^{m}\right)=H^{q}\left(K_{r}^{\prime} ; \mathscr{O} \oplus \mathscr{G}\right) \\
& =H^{q}\left(K_{r}^{\prime} ; \mathscr{O}\right) \oplus H^{q}\left(K_{r}^{\prime} ; \mathscr{G}\right) .
\end{aligned}
$$

So $H^{q}\left(K_{r}^{\prime} ; \mathscr{O}\right)=0$ for $q>0$ and $K_{r}^{\prime}$ is holomorphically convex ([6] page 73). We conclude by invoking Lemma 2.

3. A duality theorem. Choose a domain $D$ in $X$ fulfilling (a) and (b), a locally finite partition $\left\{K_{n}\right\}_{n \in \mathbf{N}}$ of $S$ by holomorphically convex compact sets and a coherent sheaf $\mathscr{F}$ on $D^{\prime}$. We consider the ring $\Gamma_{k}(S ; \mathscr{F})$ of the sections of $\mathscr{F}_{\mid S}$ with compact support. There is a canonical isomorphism between $\Gamma_{k}(S ; \mathscr{F})$ and $\sum_{n} \Gamma\left(K_{n} ; \mathscr{F}\right)$. We can consider the inductive limit topology on $\Gamma\left(K_{n} ; \mathscr{F}\right)$, the direct sum topology on $\sum_{n} \Gamma\left(K_{n} ; \mathscr{F}\right)$ and the induced topology on $\Gamma_{k}(S ; \mathscr{F})$. This is a D.F.S. topology which does not depend on the partition that we have chosen. In fact if $K$ is a compact subset of $D^{\prime}$ and $H_{1}, \ldots, H_{m}$ a partition of $K$ by compact sets, then the inductive limit topology on $\Gamma(K ; \mathscr{F})$ is the direct sum of the inductive limit topologies on $\Gamma\left(H_{i} ; \mathscr{F}\right)$. Therefore if 
$\left\{K_{s}\right\}_{s \in \mathbf{N}}$ and $\left\{K_{l}^{\prime}\right\}_{l \in \mathbf{N}}$ are two partitions we have:

$$
\begin{aligned}
\Gamma_{k}(S ; \mathscr{F}) & =\sum_{s \in \mathbf{N}} \Gamma\left(K_{s} ; \mathscr{F}\right)=\sum_{s \in \mathbf{N}} \sum_{l} \Gamma\left(K_{s} \cap K_{l}^{\prime} ; \mathscr{F}\right) \\
& =\sum_{l} \sum_{s} \Gamma\left(K_{s} \cap K_{l}^{\prime} ; \mathscr{F}\right)=\sum_{l} \Gamma\left(K_{l}^{\prime} ; \mathscr{F}\right) .
\end{aligned}
$$

This topology on $\Gamma_{k}(S ; \mathscr{F})$ will be referred to as the natural topology.

Now consider the sequence:

$$
\cdots \rightarrow H_{S}^{q}\left(D^{\prime} ; \mathscr{F}\right) \rightarrow H^{q}\left(D^{\prime} ; \mathscr{F}\right) \rightarrow H^{q}(D ; \mathscr{F}) \rightarrow H_{S}^{q+1}\left(D^{\prime} ; \mathscr{F}\right) \rightarrow \cdots
$$

with the topology derived from its short exact sequence of F.S. space.

REMARK 1. If $r: \Gamma\left(D^{\prime} ; \mathscr{F}\right) \rightarrow \Gamma(D ; \mathscr{F})$ denotes the restriction, we have the following topological isomorphisms:

$$
\begin{aligned}
& H_{S}^{0}\left(D^{\prime} ; \mathscr{F}\right) \sim \operatorname{Ker} r \\
& H_{S}^{1}\left(D^{\prime} ; \mathscr{F}\right) \sim \operatorname{CoKer} r \\
& H_{S}^{q}\left(D^{\prime} ; \mathscr{F}\right) \sim H^{q-1}(D ; \mathscr{F}) \quad \text { if } q \geq 2 .
\end{aligned}
$$

It follows from Lemma 1.

In this section we want to prove:

THEOREM 4. If $D$ fulfills (a) and (b) then for every $q \geq 0, H_{S}^{q}\left(D^{\prime} ; \mathscr{F}\right)$ is F.S. and its dual is topologically isomorphic to $\Gamma_{k}\left(S ; \operatorname{Ext}^{n-q}\left(\mathscr{F} ; \Omega^{n}\right)\right)$ with the natural topology.

To prove this theorem we need to make some other remarks. Let $U$ be a Stein neighborhood of a holomorphically convex compact set $K \subseteq X$. For $q \geq 0$ the inductive limit topology on $\Gamma\left(K ; \operatorname{Ext}^{q}\left(\mathscr{F} ; \Omega^{n}\right)\right)$ induces a D.F.S. topology on $\operatorname{Ext}^{q}\left(K ; \mathscr{F} ; \Omega^{n}\right)$ via the canonical isomorphism.

We will call this the $L$ topology.

On the other hand, Theorems 1 and 2 of $\S 2$ imply that $H_{K}^{q}(U ; \mathscr{F})$ is F.S. for every $q \geq 0$, then by $(\gamma)$, $\operatorname{Ext}^{q}\left(K ; \mathscr{F} ; \Omega^{n}\right)$ with the topology of the sequence (2.3) is D.F.S. We will call this the $T$ topology.

LEMMA 5. The $T$ and $L$ topologies coincide on $\operatorname{Ext}^{q}\left(K ; \mathscr{F} ; \Omega^{n}\right)$ for every $q \geq 0$.

Proof. If $q=0$, this is just Lemma 4. If $\mathscr{F}$ is locally free, then $\operatorname{Ext}^{q}\left(K ; \mathscr{F} ; \Omega^{n}\right)=\Gamma\left(K ; \operatorname{Ext}^{q}\left(\mathscr{F} ; \Omega^{n}\right)\right)=0$ for every $q>0$ and the lemma is proved. If $\mathscr{F}$ is coherent, by excision the $T$ topology on 
$\operatorname{Ext}^{q}\left(K ; \mathscr{F} ; \Omega^{n}\right)$ does not depend on the choice of the Stein neighborhood of $K$. The proof is again by induction on $S(\mathscr{F})=n-\operatorname{prof} \mathscr{F}$.

Consider a sequence $0 \rightarrow \mathscr{G} \stackrel{\lambda}{\rightarrow} \mathscr{O}^{r} \stackrel{\mu}{\rightarrow} \mathscr{F} \rightarrow 0$ on a Stein neighborhood $W$ of $K$ in $U$, with $S(\mathscr{G}) \leq S(\mathscr{F})-1$. We derive:

$$
\begin{aligned}
0 \rightarrow \operatorname{Hom}\left(\mathscr{F} ; \Omega^{n}\right) \rightarrow & \operatorname{Hom}\left(\mathscr{O}^{r} ; \Omega^{n}\right) \rightarrow \operatorname{Hom}\left(\mathscr{G} ; \Omega^{n}\right) \stackrel{\sigma^{0}}{\longrightarrow} \\
& \operatorname{Ext}^{1}\left(\mathscr{F} ; \Omega^{n}\right) \rightarrow 0
\end{aligned}
$$

and the isomorphisms $\sigma^{p-1}: \operatorname{Ext}^{p-1}\left(\mathscr{G} ; \Omega^{n}\right) \rightarrow \operatorname{Ext}^{p}\left(\mathscr{F} ; \Omega^{n}\right)$ when $p \geq$ 2. Since $K$ is holomorphically convex (3.1) induces:

$$
\begin{aligned}
0 \rightarrow \operatorname{Hom}\left(K ; \mathscr{F} ; \Omega^{n}\right) \rightarrow \operatorname{Hom}\left(K ; \mathscr{O}^{r} ; \Omega^{n}\right) \rightarrow \operatorname{Hom}\left(K ; \mathscr{G} ; \Omega^{n}\right) \\
\stackrel{\tilde{\sigma}^{0}}{\rightarrow} \operatorname{Ext}^{1}\left(K ; \mathscr{F} ; \Omega^{n}\right) \rightarrow 0
\end{aligned}
$$

and the isomorphisms: $\tilde{\sigma}^{p-1}: \operatorname{Ext}^{p-1}\left(K ; \mathscr{G} ; \Omega^{n}\right) \rightarrow \operatorname{Ext}^{p}\left(K ; \mathscr{F} ; \Omega^{n}\right)$ when $p \geq 2$.

If the spaces have the $L$ topologies all of these are topological homomorphisms.

Since the lemma is proved for $p=0$, for the case $p=1$ it is enough to prove that $\tilde{\sigma}^{0}$ is $T$ continuous. Consider the commutative diagram:

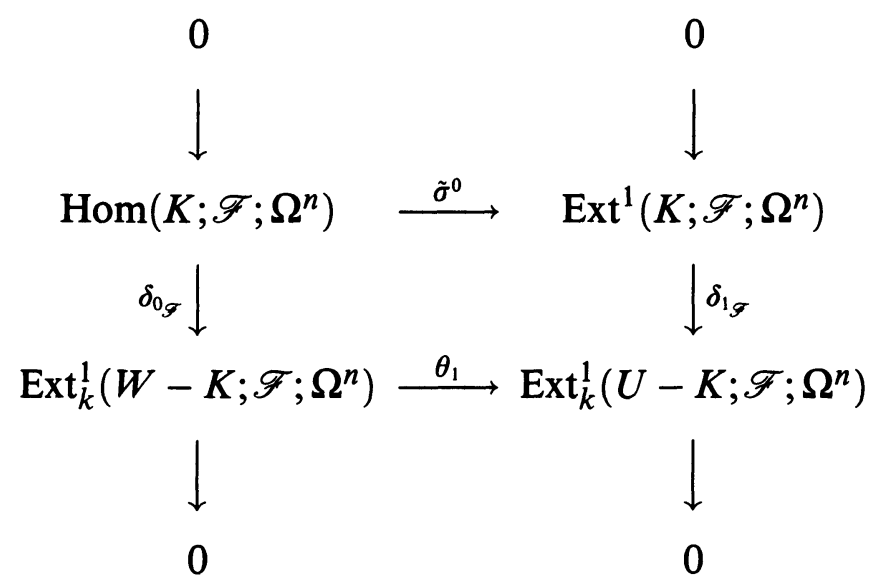

where $\delta_{0_{g}}$ and $\delta_{1_{g}}$ are those in (2.3) and $\theta_{1}$ is that in (2.6).

Since $\delta_{1_{\mathcal{F}}}$ is a topological isomorphism and $\theta_{1} \circ \delta_{0_{\mathcal{F}}}$ is continuous, it follows that $\tilde{\sigma}^{0}$ is continuous.

If $2 \leq p \leq n-1$, by induction hypothesis the $L$ and $T$ topologies coincide on $\operatorname{Ext}^{p-1}\left(K ; \mathscr{G} ; \Omega^{n}\right)$, so we must prove the $\tilde{\sigma}^{p-1}$ is $T$ continuous. 
$W$ is Stein, so $\operatorname{Ext}_{k}^{p}\left(W ; \mathscr{F} ; \Omega^{n}\right)=0, \operatorname{Ext}_{k}^{p+1}\left(W ; \mathscr{F} ; \Omega^{n}\right)$ is D.F.S. and we have the commutative diagram:

$$
\begin{aligned}
& \operatorname{Ext}^{p-1}\left(K ; \mathscr{G} ; \Omega^{n}\right) \stackrel{\delta_{p-1 \mathscr{G}}}{\longrightarrow} \operatorname{Ext}_{k}^{p}\left(W-K ; \mathscr{G} ; \Omega^{n}\right) \\
& \downarrow \tilde{\sigma}^{p-1} \\
& 0 \rightarrow \operatorname{Ext}^{p}\left(K ; \mathscr{F} ; \Omega^{n}\right) \stackrel{\delta_{p_{\mathscr{F}}}}{\longrightarrow} \operatorname{Ext}_{k}^{p+1}\left(W-K ; \mathscr{F} ; \Omega^{n}\right)
\end{aligned}
$$

$\delta_{p_{\mathscr{F}}}$ is an injective topological homomorphism, the map $\delta_{p_{\mathcal{F}}} \circ \tilde{\sigma}^{p-1}=$ $\theta_{p} \circ \delta_{p-1_{g}}$ is continuous, then $\tilde{\sigma}^{p-1}$ is continuous. If $p=n$, we will prove that $\operatorname{Ext}^{n}\left(K ; \mathscr{F} ; \Omega^{n}\right)$ is finite dimensional. There is a unique D.F.S. topology on a finite dimensional vector space, so the $L$ and $T$ topologies must coincide.

If prof $\mathscr{F} \geq 1$ then $\operatorname{Ext}^{n}\left(K ; \mathscr{F} ; \Omega^{n}\right)=0$.

If prof $\mathscr{F}=0$, we take the sequence:

$$
0 \rightarrow \mathscr{H}_{A}^{0}(\mathscr{F}) \rightarrow \mathscr{F} \rightarrow \mathscr{G} \rightarrow 0 \text { on } U,
$$

where $\left.\mathscr{H}_{A}^{0}(\mathscr{F})\right|_{U-A}=0, A$ is discrete in $U$ and prof $\mathscr{G} \geq 1$. From this we derive:

$\rightarrow \operatorname{Ext}^{n}\left(K ; \mathscr{G} ; \Omega^{n}\right) \rightarrow \operatorname{Ext}^{n}\left(K ; \mathscr{F} ; \Omega^{n}\right) \rightarrow \operatorname{Ext}^{n}\left(K ; \mathscr{H}_{A}^{0}(\mathscr{F}) ; \Omega^{n}\right) \rightarrow \cdots$.

Since prof $\mathscr{G} \geq 1, \operatorname{Ext}^{n}\left(K ; \mathscr{G} ; \Omega^{n}\right)=0$ and it is enough to prove that $\operatorname{Ext}^{n}\left(K ; \mathscr{H}_{A}^{0}(\mathscr{F}) ; \Omega^{n}\right)=\operatorname{Ext}^{n}\left(K \cap A ; \mathscr{H}_{A}^{0}(\mathscr{F}) ; \Omega^{n}\right)$ is finite dimensional. The set $A$ is discrete in $U$, hence $A \cap K=\left\{x_{1}, \ldots, x_{m}\right\}$, then

$$
\operatorname{Ext}^{n}\left(K \cap A ; \mathscr{H}_{A}^{0}(\mathscr{F}) ; \Omega^{n}\right)=\sum_{i=1}^{m} \operatorname{Ext}^{n}\left(\left\{x_{i}\right\} ; \mathscr{H}_{A}^{0}(\mathscr{F}) ; \Omega^{n}\right) .
$$

For every $i$ with $1 \leq i \leq m$, let $U_{i}$ be an open neighborhood of $x_{i}$ such that $\left.\operatorname{Ext}^{n}\left(\mathscr{H}_{A}^{0}(\mathscr{F}) ; \Omega^{n}\right)\right|_{U_{i}-\left\{x_{i}\right\}}=0$. If $x_{i} \in V \subseteq U_{i}$, then

$$
\begin{aligned}
& \Gamma\left(V ; \operatorname{Ext}^{n}\left(\mathscr{H}_{A}^{0}(\mathscr{F}) ; \Omega^{n}\right)\right)=\Gamma\left(U_{i} ; \operatorname{Ext}^{n}\left(\mathscr{H}_{A}^{0}(\mathscr{F}) ; \Omega^{n}\right)\right) ; \text { so } \\
& \Gamma\left(U_{i} ; \operatorname{Ext}^{n}\left(\mathscr{H}_{A}^{0}(\mathscr{F}) ; \Omega^{n}\right)\right) \\
& \quad=\underset{V \ni x_{i}}{\lim } \Gamma\left(V ; \operatorname{Ext}^{n}\left(\mathscr{H}_{A}^{0}(\mathscr{F}) ; \Omega^{n}\right)\right) \\
& \left.=\Gamma\left(\left\{x_{i}\right\} ; \operatorname{Ext}^{n}\left(\mathscr{H}_{A}^{0}(\mathscr{F}) ; \Omega^{n}\right)\right)=\operatorname{Ext}^{n}\left(\left\{x_{i}\right\} ; \mathscr{H}_{A}^{0}(\mathscr{F}) ; \Omega^{n}\right)\right) .
\end{aligned}
$$

Therefore $\Gamma\left(U_{i} ; \operatorname{Ext}^{n}\left(\mathscr{H}_{A}^{0}(\mathscr{F}) ; \Omega^{n}\right)\right)$ is F.S. and D.F.S., it follows that it is finite dimensional.

Proof of Theorem 4. The space $H_{S}^{q}\left(D^{\prime} ; \mathscr{F}\right)$ is F.S. for every $q \geq 0$ by Theorems 1 and 2. Moreover if $S$ is compact the theorem follows from Lemma 5. 
If $S$ is noncompact and $\left\{K_{s}\right\}_{s \in \mathbf{N}}$ is a locally finite partition of $S$ by holomorphically convex compact sets, then:

$$
\begin{aligned}
& {\left[H_{S}^{q}\left(D^{\prime} ; \mathscr{F}\right]^{\prime} \sim\left[\prod_{s \in \mathbf{N}} H_{K_{s}}^{q}(S ; \mathscr{F})^{\prime}\right]\right.} \\
& \quad=\sum_{s \in \mathbf{N}}\left[H_{K_{s}}^{q}(S ; \mathscr{F})\right]^{\prime} \sim \sum_{s \in \mathbf{N}} \Gamma\left(K_{s}: \operatorname{Ext}^{n-q}\left(\mathscr{F} ; \Omega^{n}\right)\right) \\
& \quad=\Gamma_{k}\left(S ; \operatorname{Ext}^{n-q}\left(\mathscr{F} ; \Omega^{n}\right)\right) \text { by definition. }
\end{aligned}
$$

4. Cohomology with compact support. Let $D$ be a domain in $X$ fulfilling conditions (a) and (b), let $\mathscr{F}$ be a coherent sheaf on $D^{\prime}$, then we have the following:

THEOREM 5. The cohomology groups $H_{k}^{q}(D ; \mathscr{F})$ with compact support are D.F.S. for every $q \geq 0$. If $\mathscr{F}$ is locally free then $H_{k}^{q}(D ; \mathscr{F})=0$ for $q \neq 1$ and $q \neq n$.

Proof. Consider the exact sequence of sheaves on $D^{\prime}$ :

$$
0 \rightarrow \mathscr{F}_{D} \rightarrow \mathscr{F} \rightarrow \mathscr{F}_{S} \rightarrow 0
$$

and the corresponding exact cohomology sequence with compact support:

$$
\begin{aligned}
0 & \rightarrow T_{k}(D ; \mathscr{F}) \rightarrow \Gamma_{k}\left(D^{\prime} ; \mathscr{F}\right) \rightarrow \Gamma_{k}(S ; \mathscr{F}) \stackrel{m_{0}}{\rightarrow} H_{k}^{1}(D ; \mathscr{F}) \\
& \rightarrow H_{k}^{1}\left(D^{\prime} ; \mathscr{F}\right) \rightarrow H_{k}^{1}(S ; \mathscr{F}) \rightarrow \cdots \rightarrow H_{k}^{q-1}(S ; \mathscr{F}) \\
& \rightarrow H_{k}^{q}(D ; \mathscr{F}) \rightarrow H_{k}^{q}\left(D^{\prime} ; \mathscr{F}\right) \rightarrow H_{k}^{q}(S ; \mathscr{F}) \rightarrow \cdots
\end{aligned}
$$

with the topology induced by its short exact sequence of D.F.S. spaces ([1] page 25 ).

Let $\left\{K_{n}\right\}_{n \in \mathbf{n}}$ be a locally finite partition of $S$ by holomorphically convex compact sets, then for $q \geq 1$ we have:

$$
H_{k}^{q}(S ; \mathscr{F}) \sim \sum_{r} H^{q}\left(K_{r} ; \mathscr{F}\right)=0 .
$$

It follows that for $q \geq 2$ the space $H_{k}^{q}(D ; \mathscr{F})$ is isomorphic to the D.F.S. space $H_{k}^{q}\left(D^{\prime} ; \mathscr{F}\right)$ [2]. Since $\Gamma_{k}(D ; \mathscr{F})$ is always D.F.S. it remains to prove that $H_{k}^{1}(D ; \mathscr{F})$ also is. At this end, suppose first that prof $\mathscr{F} \geq 1$, then

$$
\Gamma_{k}\left(D^{\prime} ; \mathscr{F}\right) \sim\left[\operatorname{Ext}^{n}\left(D^{\prime} ; \mathscr{F} ; \Omega^{n}\right)\right]^{\prime} \sim\left[\Gamma\left(D^{\prime} ; \operatorname{Ext}^{n}\left(\mathscr{F} ; \Omega^{n}\right)\right)\right]^{\prime}=0
$$


and the map $m_{0}$ is injective. Since $H_{k}^{1}\left(D^{\prime} ; \mathscr{F}\right)$ is D.F.S. the image of $m_{0}$ is closed and by Lemma 1 it is a topological homomorphism.

Moreover it is enough to prove that $\operatorname{Im} m_{0}$ with the topology induced by $H_{k}^{1}(D ; \mathscr{F})$ is D.F.S. (in fact it contains the closure of 0 ). However $\operatorname{Im} m_{0}$ is topologically isomorphic to $\Gamma_{k}(S ; \mathscr{F})$ which is D.F.S. since it is the zero cohomology group of a complex of D.F.S. spaces. If prof $\mathscr{F}=0$ we have the exact sequence (2.7) and the corresponding:

$$
\begin{aligned}
\cdots & \rightarrow H_{k}^{1}\left(D ; \mathscr{H}_{A}^{0}(\mathscr{F})\right) \rightarrow H_{k}^{1}(D ; \mathscr{F}) \rightarrow H_{k}^{1}(D ; \mathscr{G}) \\
& \rightarrow H_{k}^{2}\left(D ; \mathscr{H}_{A}^{0}(\mathscr{F})\right) \rightarrow \cdots
\end{aligned}
$$

Since $\mathscr{H}_{A}^{0}(\mathscr{F})$ has discrete support we have:

$$
H_{k}^{1}\left(D ; \mathscr{H}_{A}^{0}(\mathscr{F})\right)=H_{k}^{2}\left(D ; \mathscr{H}_{A}^{0}(\mathscr{F})\right)=0 ;
$$

so the spaces $H_{k}^{1}(D ; \mathscr{F})$ and $H_{k}^{1}(D ; \mathscr{G})$ are isomorphic. Now prof $\mathscr{G} \geq$ 1 and the theorem follows from the preceding step. If $\mathscr{F}$ is locally free the theorem follows from Theorem 1 and duality.

5. The two-dimensional case. If the manifold $X$ has complex dimension 2 then we obtain:

THEOREM 6. Let $D$ be a domain in a connected Stein manifold $X$ of complex dimension 2, $\mathscr{L}$ a locally free nonzero sheaf on $X$, then: $H^{1}(D ; \mathscr{L})$ is F.S. if and only if $H_{k}^{2}(D ; \mathscr{L})$ is D.F.S. if and only if $D$ fulfills (a) and (b).

Proof. If $D$ fulfills (a) and (b) then $H^{1}(D ; \mathscr{L})$ is F.S. by Theorems 1 and 5 . Conversely as in Theorem 3 we can prove that $D$ fulfills (b) and that $H^{1}\left(D^{\prime} ; \mathscr{L}\right)=0$. To prove (a) we choose a point $p \in \partial D^{\prime}$ and an open Stein neighborhood $U$ of $p$ such that $\left.\mathscr{L}\right|_{U}=\mathscr{O}^{r}$. We have the Mayer-Vietoris sequence:

$$
\begin{aligned}
\cdots & \rightarrow H^{1}\left(D^{\prime} ; \mathscr{L}\right) \oplus H^{1}(U ; \mathscr{L}) \rightarrow H^{1}\left(D^{\prime} \cap U ; \mathscr{L}\right) \\
& \rightarrow H^{2}\left(D^{\prime} \cup U ; \mathscr{L}\right) \rightarrow \cdots
\end{aligned}
$$

Now $U$ is Stein and $X$ is two-dimensional, then:

$$
H^{1}\left(D^{\prime} ; \mathscr{L}\right)=H^{1}(U ; \mathscr{L})=H^{2}\left(D^{\prime} \cup U ; \mathscr{L}\right)=0 .
$$

So

$$
H^{1}\left(D^{\prime} \cap U ; \mathscr{L}\right) \sim\left[H^{1}\left(D^{\prime} \cap U ; \mathscr{O}\right)\right]^{r}=0
$$


Then $D^{\prime} \cap U$ is Stein ([6] page 65). The domain $D^{\prime}$ is a locally Stein manifold, then a Stein manifold. However $H_{k}^{2}(D ; \mathscr{L})$ is D.F.S. if and only if $H^{1}\left(D ; \operatorname{Hom}\left(\mathscr{L} ; \Omega^{2}\right)\right)$ is F.S. if and only if $D$ fulfills (a) and (b).

Corollary 1. Let $X$ be a two-dimensional connected Stein manifold and $D$ a domain in $X$; suppose that there exists a nonzero locally free sheaf $\mathscr{L}_{0}$ in $X$ such that $H^{1}\left(D ; \mathscr{L}_{0}\right)$ is F.S. or $H_{k}^{2}\left(D ; \mathscr{L}_{0}\right)$ is D.F.S. Let $\mathscr{F}$ be a coherent sheaf on $D^{\prime}$, then:

(1) The groups $H_{S}^{q}(D ; \mathscr{F})$ are F.S. for every $q \geq 0$ and their dual spaces are topologically isomorphic to $\Gamma_{k}\left(S ; \operatorname{Ext}^{2-q}\left(\mathscr{F} ; \Omega^{2}\right)\right)$ with the natural topology.

(2) The groups $H_{k}^{q}(D ; \mathscr{F})$ are D.F.S. for every $q \geq 0$.

Proof. It follows from Theorems 4, 5 and 6.

\section{REFERENCES}

[1] Andreotti-Banica, Relative duality on complex spaces, Revue Roum. de Math., 9 (1975), 9 (1976).

[2] Andreotti-Kas, Duality on complex spaces, Ann. Sc. Nor. Pisa, 27 (1973), 187263.

[3] Banica-Stanasila, Methodes Algebrique dans la Theorie Globale des Espaces Complexes, Paris, Gouthier Villars, 1977.

[4] Cassa, Coomologia separata sulle varietà complesse, Ann. Sc. Nor. Pisa, 25 (1971), 291-323.

[5] The cohomology of an exhaustible complex space, Bollettino U.M.I., (6) 4 B, (1985), 321-341.

[6] Coen, Annullation de la cohomologie..., Composition Mathematica, 37 (1978), 63-75.

[7] Godement, Topologie Algebrique et Theorie des Faissceaux, Herman, Paris, 1958.

[8] Grauert-Remmert, Theory of Stein Spaces, Springer-Verlag, Berlin, Heidelberg, New York, 1979.

[9] Siu, Analytic sheaf cohomology groups. .., Trans. Amer. Math. Soc., 143 (1969), 77-94.

[10] Pseudoconvexity and the problem of Levi, Bull. Amer. Math. Soc., 84, (1978), 317-326.

[11] Trapani, Inviluppi di olomorfia e gruppi di coomologia di Hausdorff, Rendiconti del seminario matematico dell'Università di Padova, Vol. LXXV (1986).

[12] Coomologia di Hausdorff e forma di Levi, Annali di matematica pura ed applicata, Serie IV, Tomo CXLIV (1986).

[13] Wells, Compact holomorphically convex subsets of a Stein manifold, Trans. Amer. Math. Soc., 136 (1969), 509-516.

[14] Differential Analysis on Complex Manifolds, Springer-Verlag, New York, Heidelberg, Berlin 1979. 
[15] Zame, Holomorphic convexity of compact sets in analytic spaces and the structure of algebras of holomorphic forms, Trans. Amer. Math. Soc., 222 (1976), 107127.

Received November 10, 1986.

Università di Pisa

VIA BUONARROTI 2

56100 PISA, ITALY 


\section{PACIFIC JOURNAL OF MATHEMATICS EDITORS}

V. S. VARADARAJAN (Managing Editor) University of California Los Angeles, CA 90024

HeRbert Clemens

University of Utah

Salt Lake City, UT 84112

R. FINN

Stanford University

Stanford, CA 94305
ROBION KIRBY

University of California

Berkeley, CA 94720

C. C. MOORE

University of California

Berkeley, CA 94720

HAROLD STARK

University of California, San Diego

La Jolla, CA 92093

\section{ASSOCIATE EDITORS}
R. ARENS
E. F. BECKENBACH
B. H. NEUMANN
F. Wolf
K. YOSHIDA (1906-1982)

\section{SUPPORTING INSTITUTIONS}

UNIVERSITY OF ARIZONA UNIVERSITY OF OREGON

UNIVERSITY OF BRITISH COLUMBIA UNIVERSITY OF SOUTHERN CALIFORNIA

CALIFORNIA INSTITUTE OF TECHNOLOGY UNIVERSITY OF CALIFORNIA MONTANA STATE UNIVERSITY STANFORD UNIVERSITY

UNIVERSITY OF NEVADA, RENO UNIVERSITY OF HAWAII NEW MEXICO STATE UNIVERSITY UNIVERSITY OF TOKYO UNIVERSITY OF UTAH OREGON STATE UNIVERSITY WASHINGTON STATE UNIVERSITY UNIVERSITY OF WASHINGTON 


\section{Pacific Journal of Mathematics}

Vol. 134, No. $1 \quad$ May, 1988

Marco Abate, Annular bundles . ............................

Ralph Cohen, Wen Hsiung Lin and Mark Mahowald, The Adams

spectral sequence of the real projective spaces

Harry Joseph D'Souza, Threefolds whose hyperplane sections are elliptic surfaces .........................................57

Theodore Gerard Faticoni, Localization in finite-dimensional FPF rings . . . 79

Daniel Hitt, Invariant subspaces of $\mathscr{H}^{2}$ of an annulus ................ 101

Ellen Kirkman and James J. Kuzmanovich, On the global dimension of fibre products

Angel Rafael Larotonda and Ignacio Zalduendo, Homogeneous spectral sets and local-global methods in Banach algebras

Halsey Lawrence Royden, Jr., Invariant subspaces of $\mathscr{H}^{p}$ for multiply

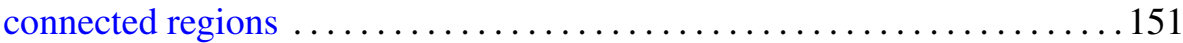

Jane Sangwine-Yager, A Bonnesen-style inradius inequality in 3-space . . . . 173

Stefano Trapani, Holomorphically convex compact sets and cohomology

Thomas Vogel, Uniqueness for certain surfaces of prescribed mean

curvature 